\title{
Comparative Statics of Oligopoly Equilibrium in a Pure Exchange Economy
}

\author{
Somdeb Lahiri \\ SPM, PD Petroleum University, Gujarat, India \\ E-mail: somdeb.lahiri@yahoo.co.in \\ Received January 25, 2011; revised March 1, 2011; accepted March 29, 2011
}

\begin{abstract}
In this paper we consider an oligopoly and we are concerned with the effect of entry in the market of buyers and/or sellers on the price of the good being sold and the pay-offs/utilities of the buyers and sellers. The major results obtained in this paper are the following: 1) Given the number of buyers both individual as well as aggregate offers go up as the number of sellers increases. Further, the price of $\mathrm{Y}$ decreases, each buyer is better off and each seller is worse off as the number of sellers increases. 2) Given the number of sellers, the price of $\mathrm{Y}$ increases, each buyer is worse off and each seller is better off as the number of buyers increases. 3) As the economy is replicated the equilibrium price decreases. The sequence of equilibrium prices thus obtained converges to the competitive equilibrium price of the original economy. 4) As the economy is replicated the buyers are better off. The sequence of consumption bundles of the buyers converges to the consumption bundle of the buyers at the competitive equilibrium of the original economy. 5) As the economy is replicated the sellers are worse off. The sequence of consumption bundles of the sellers converges to the consumption bundle of the sellers at the competitive equilibrium of the original economy.
\end{abstract}

Keywords: Oligopoly, Pure Exchange, Comparative Statics, Entry-Exit, Replication

\section{Introduction}

We consider a general equilibrium model with two goods, i.e. money $(\mathrm{X})$ and an infinitely divisible good $(\mathrm{Y})$, where buyers are initially endowed only with $\mathrm{X}$ and sellers are initially endowed only with Y. Unlike the perfectly competitive model we assume that sellers behave strategically. However where buyers are concerned, they may or may not behave strategically. If buyers also behave strategically then we have a simple version of a strategic market game due to Shubik [1], Shapley [2] and Shapley and Shubik [3] that is discussed in Gabszewicz and Michel [4]. The latter class of models has been studied in [5]. If they act as price-takers then the pure exchange economy becomes a version of Counot's model of oligopoly. Such models have been discussed in [6].

In this paper we consider an oligopoly and we are concerned with the effect of entry in the market of buyers and/or sellers on the price of $\mathrm{Y}$ and the payoffs/utilities of the buyers and sellers. An increase in the number of buyers leads to an increase in the availability of $X$ as also an increase in the demand for $\mathrm{Y}$; an increase in the number of sellers leads to an increase in the availability of $\mathrm{Y}$ as also an increase in the demand for $\mathrm{X}$. Hence our paper is concerned with comparative statics in oligopolistic markets. Amir and Bloch [7] discuss these issues in a very general model for bilateral oligopolies. We discuss similar issues for an oligopoly assuming that buyers and sellers have Cobb-Douglas utility functions. Hence whereas our buyers are price takers in the Cournotian tradition, in the kind of bilateral oligopoly that Amir and Bloch [7] deal with, buyers behave strategically and submit bids against the offers that sellers place at the trading post. Thus the two market structures are entirely different. Further our use of Cobb-Douglas utility functions allows us to obtain sharper results than what Amir and Bloch [7] do, albeit in a different market structure. The analysis in Amir and Bloch [7] "focuses on three questions: When does an increase in the number of buyers result in an increase in the total bids on the market? In an increase in the equilibrium price of the private good? In an increase in the equilibrium utility of the sellers?” We address all the above questions and obtain definite answers for the same; in addition we show that entry of new buyers lead to a decline in the utility of existing buyer. We also obtain definite answers for the ef- 
fect of the entry of sellers on the total and individual offers, the price of $\mathrm{Y}$ and the utility of both buyers and sellers. Our model is somewhat more general than the pioneering version due to Codognato and Gabszewicz [6].

While we do state results for the asymptotic convergence of prices and allocations when we keep replicating the economy, these results are along the lines established in [8] or [9]. Such price-allocation pairs converge to the unique competitive equilibrium of the non-replicated original economy as should have been anticipated. However, the monotonic behavior of prices, consumption bundles and utilities that we observe under both onesided entry as well as with replication of the economy is something that is beyond the scope of the general framework in which the asymptotic analysis is carried out. In particular we are able to go beyond the questions addressed in earlier investigations for bilateral oligopoly and claim that buyers are unconditionally better off and sellers are unconditionally worse off as the economy is replicated. This result may not be true for a bilateral oligopoly.

The assumption that all utility functions are CobbDouglas is in the present context hardly a serious limitation of the paper. First Cobb-Douglas or log-linear utility functions are very common (if not mandatory) in applied general equilibrium analysis. While the functional form is specific there is considerable flexibility in the choice of the parameter which determines the exact utility function. Second a lot of deep results in general equilibrium theory (to the extent of proving that the competitive mechanism is uniquely informationally efficient) has been possible only in the case of Cobb-Douglas utility functions. Third using Cobb-Douglas utility functions for buyers is a theoretically desirable variant of using linear demand functions on which most of industrial organization is based. Downward sloping linear demand functions are generated by quasi-linear utility functions whose non-linear part is a concave quadratic function. Fourth Cobb-Douglas utility functions are the only utility functions to be characterized by the empirically relevant property that the fraction (and not the amount) of total expenditure allocated to a good is independent of prices and expenditure. Finally, the use of Cobb-Douglas utility functions rather than an arbitrary one makes the analysis in this paper accessible to a much larger audience than would be possible otherwise. Thus it can be expected that the comparative static results that we obtain in this paper will throw some light on the behavior of oligopolistic markets in the setting of pure exchange.

It is worth noting that unlike monopolistic competition, the product sold in an oligopoly is homogeneous and identical across sellers. Hence for all practical purposes, the assumption that all sellers have the same preferences and all buyers have the same preferences is also not a major limitation of the model. It just makes the analysis simpler without sacrificing any major implication.

\section{The Model}

Following Amir and Bloch [1] we consider an economy with two goods i.e. money (X) and another non-monetary divisible commodity (Y). The economy has two types of traders: buyers each of whom own one unit of $\mathrm{X}$, and sellers each of whom are endowed with one unit of $\mathrm{Y}$. Buyers are indexed by $b=1, \cdots, m$ and sellers are indexed by $s=1, \cdots, n$. Let $\mathrm{x}$ denote the quantity of money and $\mathrm{y}$ the quantity of $\mathrm{Y}$ allocated to a trader. A consumption bundle is a pair $(x, y) \in \mathrm{R}_{+}^{2}$.

We assume that all buyers have the same utility function $U: \mathrm{R}_{+}^{2} \rightarrow \mathrm{R}$ and all sellers have the same utility function $V: \mathrm{R}_{+}^{2} \rightarrow \mathrm{R}$. We further assume that the two utility functions are Cobb-Douglas.

Assumption 1: There exists $\alpha, \beta \in(0,1)$ such that $U(x, y)=x^{\alpha} y^{1-\alpha}$ and $V(x, y)=x^{\beta} y^{1-\beta}$ for all $(x, y) \in$ $\mathrm{R}_{+}^{2}$.

An allocation is a list $\left[\left(x_{b}, y_{b}\right)_{b=1, \cdots, m},\left(x_{s}, y_{s}\right)_{s=1, \cdots, n}\right]$ such that $\sum_{b=1}^{m} x_{b}+\sum_{s=1}^{n} x_{s}=m$ and $\sum_{b=1}^{m} y_{b}+\sum_{s=1}^{n} y_{s}=n$. For the sake of simplicity we shall often denote an allocation as $\left[\left(x_{b}, y_{b}\right),\left(x_{s}, y_{s}\right)\right]$.

A price $p$ is a positive real number such that $\mathrm{p}$ units of money has to be paid to purchase one unit of $\mathrm{Y}$.

A competitive equilibrium is a price allocation pair $\left(p,\left[\left(x_{b}, y_{b}\right),\left(x_{s}, y_{s}\right)\right]\right)$ such that:

1) for each $b=1, \cdots, m: x_{b}=1-p y_{b}$ and $y_{b}$ maximizes $U(1-p y, y)$ subject to $y \in[0,1]$;

2) for each $s=1, \cdots, n: x_{s}=p-p y_{s}$ and $y_{s}$ maximizes $V(p-p y, y)$ subject to $y \in[0,1]$.

Given the structure of preferences that we have assumed it is well known that at price $p$ :

1) $x_{b}=1-p y_{b}$ and $y_{b}$ maximizes $U(1-p y, y)$ subject to $y \in[0,1]$ if and only if $x_{b}=\alpha$ and $y_{b}=1-\alpha / p$;

2) $x_{s}=p-p y_{s}$ and $y_{s}$ maximizes $V(p-p y, y)$ subject to $y \in[0,1]$ if and only if $x_{s}=\beta p$ and $y_{s}=1-\beta$.

Hence:

1) $x_{b}=1-p y_{b}$ and $y_{b}$ maximizes $U(1-p y, y)$ subject to $y \in[0,1]$ if and only if $x_{b}=1-p y_{b}$ and $y_{b}$ maximizes $U(1-p y, y)$ subject to $y \in$ $(0,1)$

2) $x_{s}=p-p y_{s}$ and $y_{s}$ maximizes $V(p-p y, y)$ subject to $y \in[0,1]$ if and only if $x_{s}=p-p y_{s}$ and $y_{s}$ maximizes $V(p-p y, y)$ subject to $y \in$ $(0,1)$.

It is also clear that $x_{b}, y_{b}, x_{s}, y_{s} \in(0,1)$. 
Further $\mathrm{p}$ is a competitive equilibrium price if and only if $\sum_{b=1}^{m} x_{b}+\sum_{s=1}^{n} x_{s}=m$, i.e. $m \alpha+n \beta p=m$. Thus $p=\frac{m}{n} \frac{(1-\alpha)}{\beta}$

In this paper we depart from the competitive pricetaking behavior of the sellers and assume that each seller $s$ offers a quantity $q_{s} \in[0,1]$. We shall denote the aggregate offer $\sum_{t=1}^{m} q_{t}$ by $Q$ and the aggregate offer of all sellers other than s, i.e. $Q-q_{s}$ by $Q_{-s}$.

\section{Oligopoly Equilibrium}

Given a list of offers $\left(q_{s}\right)_{s=1, \cdots, n}$ (denoted simply as $\left(q_{s}\right)$ when there is no scope for confusion) a pair $\left[p,\left(x_{b}, y_{b}\right)_{b=1, \cdots, m}\right]$ (denoted simply as $\left[p,\left(x_{b}, y_{b}\right)\right]$ when there is no scope for confusion) is said to be a market equilibrium for $\left(q_{s}\right)$ if:

1) for each $b=1, \cdots, m: x_{b}=1-p y_{b}$ and $y_{b}$ maximizes $U(1-p y, y)$ subject to $y \in[0,1]$; and

2) $\sum_{b=1}^{m} y_{b}=\sum_{s=1}^{n} q_{s}=Q$.

It is easy to see that if $\left[p,\left(x_{b}, y_{b}\right)\right]$ is a market equilibrium for $\left(q_{s}\right)$ then:

1) $p=\frac{m(1-\alpha)}{Q}$; and

2) for each $b=1, \cdots, m: x_{b}=\alpha$ and $y_{b}=\frac{Q}{m}$.

$m(1-\alpha) / Q$ is the maximum unit price of $\mathrm{Y}$ that buyers would be willing to pay if the list of offers was $\left(q_{s}\right)$. Further the consumption bundle of each buyer under such circumstances is $\left(\alpha, \frac{Q}{m}\right)$.

Thus for a fixed aggregate offer Q, each buyer's consumption of $\mathrm{Y}$ decreases as the number of buyers i.e. $\mathrm{m}$ increases where as his consumption of money remains fixed. Consequently for a fixed aggregate offer, each existing buyer is worse off as the number of buyers increases.

If $\left(q_{s}\right)$ is the list of offers made by the sellers then the consumption bundle of seller s is $\left(\frac{m(1-\alpha)}{Q_{-s}+q_{s}} q_{s}, 1-q_{s}\right)$.

A list of offers $\left(q_{s}\right)$ is said to be an oligopoly equilibrium if for all $s=1, \cdots, n: q_{s}$ solves maximize $\mathrm{V}\left(\frac{m(1-\alpha)}{Q_{-s}+q} q, 1-q\right)$ subject to $q \in[0,1]$.

Notice that the functions $q \mid \rightarrow \frac{m(1-\alpha)}{Q_{-s}+q} q$ and

$q \mid \rightarrow 1-q$ with domain $[0,1]$ are concave. Since $V$ is strictly concave we get that $q \mid \rightarrow \mathrm{V}\left(\frac{m(1-\alpha)}{Q_{-s}+q} q, 1-q\right)$ is strictly concave as well.

Lemma 1: If $\left(q_{S}\right)$ is a oligopoly equilibrium then for all $s=1, \cdots, n$ it is the case that $0<q_{s}<1$ and $Q_{-s} \in(0, m-1)$.

Proof: Let $s=1, \cdots, n$. Thus

$\left.V\left(\frac{m(1-\alpha)}{Q_{-s}+q} q, 1-q\right)\right|_{q=1 / 2}>0=V(0,1)=V\left(\frac{m(1-\alpha)}{Q_{-s}+q}, 0\right)$

Hence the solution to maximize $V\left(\frac{m(1-\alpha)}{Q_{-s}+q} q, 1-q\right)$ subject to $q \in[0,1]$ must belong to $(0,1)$, i.e. $1>q_{s}>0$. Thus for all $t=1, \cdots, n, 0<q_{t}<1$. Thus $\mathrm{Q}_{-s} \in(0, m-1)$ for all $s=1, \cdots, n$.Q.E.D.

For $\mathrm{Q}_{-s}>0$, consider the maximization problem maximize $\mathrm{V}\left(\frac{m(1-\alpha)}{Q_{-s}+q} q, 1-q\right)$ subject to $q \in[0,1]$. As in the proof of lemma 1 , it follows that it has a solution in $(0,1)$.

Consider the equation $m(1-\alpha) \mathrm{Q}_{-s} V_{x}-\left(Q_{-s}+q\right)^{2} V_{y}$ $=0$, where $V_{x}$ (resp. $V_{y}$ ) is the partial derivative of $\mathrm{V}$ with respect to $\mathrm{x}$ (resp. y). A necessary and sufficient condition for $q \in(0,1)$ to solve the maximization problem maximize $\mathrm{V}\left(\frac{m(1-\alpha)}{Q_{-s}+q} q, 1-q\right)$ subject to $q \in[0,1]$ is that it satisfies this equation. Hence let us see whether the above system has a solution in $(0,1)$.

The above equation reduces to

$$
\begin{aligned}
& \frac{\beta Q_{-s}\left(Q_{-s}+q\right)}{q}-\frac{(1-\beta)\left(Q_{-s}+q\right)^{2}}{1-q}=0 \\
& \text { or } \beta Q_{-s}(1-q)-(1-\beta) q\left(Q_{-s}+q\right)=0 \\
& \text { or }(1-\beta) q^{2}+q Q_{-s}-\beta Q_{-s}=0 .
\end{aligned}
$$

Let us denote the solution to the above quadratic by $q_{s}\left(Q_{-s}\right)$.

Then $q_{s}\left(\mathrm{Q}_{-s}\right)=\frac{-Q_{-s} \pm \sqrt{\left(Q_{-s}\right)^{2}+4 \beta(1-\beta)}}{2(1-\beta)}$. Since $q_{s}\left(\mathrm{Q}_{-s}\right)>0$ it must be the case that

$q_{s}\left(\mathrm{Q}_{-s}\right)=\frac{-Q_{-s}+\sqrt{\left(Q_{-s}\right)^{2}+4 \beta(1-\beta)}}{2(1-\beta)}$.

Thus for all $Q_{-s}>0$, there exists a unique solution $q_{s}\left(Q_{-s}\right)$ to the problem maximize

$V\left(\frac{m(1-\alpha)}{Q_{-s}+q} q, 1-q\right)$ subject to $q \in[0,1]$ and $q_{s}\left(Q_{-s}\right)$ belongs to the open interval $(0,1)$. 
The proof of the next proposition borrows ideas from the proofs of lemmas 4 and 1 of [1].

Proposition 1: Let $\left(q_{s}\right)$ be an oligopoly equilibrium. Then $q_{s}=\frac{n-1}{n-\beta} \beta$ for all $s=1, \cdots, n$ and hence $Q=n \frac{n-1}{n-\beta} \beta$. Conversely if $q_{s}=\frac{n-1}{n-\beta} \beta$ for all $s=1, \cdots, n$ then $\left(q_{s}\right)$ is an oligopoly equilibrium. Both are independent of ' $m$ ' and ' $\alpha$ ' and both go up as $n$ (the number of sellers) increases.

Proof: Consider the function $f:[0, m-1] \rightarrow[0, m]$ defined by $f\left(Q_{-s}\right)=Q_{-s}+q\left(Q_{-s}\right)$ for all $Q_{-s}$ belonging to $[0, m-1]$. The function is well defined since we know that $q_{s}\left(Q_{-s}\right) \in(0,1)$ for all $Q_{-s} \in[0, m-1]$.

We know that $(1-\beta) q^{2}+q Q_{-s}-\beta Q_{-s}=0$ for all $Q_{-s} \in[0,1]$. Differentiating this expression with respect to $Q_{\text {-s }}$ gives us

$$
2(1-\beta) q\left(Q_{-s}\right) \frac{\mathrm{d} q}{\mathrm{~d} Q_{-s}}+q\left(Q_{-s}\right)+Q_{-s} \frac{\mathrm{d} q}{\mathrm{~d} Q_{-s}}-\beta=0
$$

Thus

$$
\frac{d q}{d Q_{-s}}=\frac{\beta-q\left(Q_{-s}\right)}{2(1-\beta) q\left(Q_{-s}\right)+Q_{-s}} .
$$

Hence

$$
\begin{aligned}
& f^{\prime}\left(Q_{-s}\right)=1+\frac{\beta-q\left(Q_{-s}\right)}{2(1-\beta) q\left(Q_{-s}\right)+Q_{-s}} \\
& =\frac{2(1-\beta) q\left(Q_{-s}\right)+Q_{-s}+\beta-q\left(Q_{-s}\right)}{2(1-\beta) q\left(Q_{-s}\right)+Q_{-s}} \\
& =\frac{(1-\beta) q\left(Q_{-s}\right)+\beta\left(1-q\left(Q_{-s}\right)\right)+\left(q\left(Q_{-s}\right)+Q_{-s}\right)}{2(1-\beta) q\left(Q_{-s}\right)+Q_{-s}}>0
\end{aligned}
$$

since the denominator is positive and each term in the numerator is positive.

Thus $f($.$) is an increasing function of Q_{-s}$.

Towards a contradiction suppose $\left(q_{s}\right)$ is an oligopoly equilibrium with $q_{s} \neq q_{t}$ for some $s, t \in\{1, \cdots, n\}$. Thus $Q_{-s} \neq Q_{-t}$ although $f\left(Q_{-s}\right)=f\left(Q_{-t}\right)$. This contradicts that $\mathrm{f}$ is strictly increasing.

Hence $q_{s}=q_{t}$ for all $s, t \in\{1, \cdots, n\}$.

Let $Q$ denote the aggregate offers at the oligopoly equilibrium. Then $q_{s}=Q / n$ for all $s=1, \cdots, n$

Thus,

$$
\begin{gathered}
(1-\beta)\left(\frac{Q}{n}\right)^{2}+\frac{Q}{n} \frac{(n-1) Q}{n}-\beta \frac{(n-1) Q}{n}=0 \\
\quad \text { or }(1-\beta) \frac{Q}{n}+\frac{(n-1) Q}{n}=\beta(n-1) .
\end{gathered}
$$

Hence

$$
\frac{Q}{n}=\frac{n-1}{n-\beta} \beta \text { and so } q_{s}=\frac{n-1}{n-\beta} \beta \text { for } s=1, \cdots, n \text {. }
$$

Conversely suppose $q_{s}=\frac{n-1}{n-\beta} \beta$ for $s=1, \cdots, n$.

Then for $s=1, \cdots, n$, maximizes $V\left(\frac{m(1-\alpha)}{Q_{-s}+q} q, 1-q\right)$

subject to $q \in[0,1]$ if and only if

$$
\frac{\beta Q_{-s}\left(Q_{-s}+q_{s}\right)}{q_{s}}-\frac{(1-\beta)\left(Q_{-S}+q_{S}\right)^{2}}{1-q_{s}}=0
$$

where $\mathrm{Q}_{-s}=\frac{(n-1)^{2}}{n-\beta} \beta$.

Thus $q_{s}$ maximizes $V\left(\frac{m(1-\alpha)}{Q_{-s}+q} q, 1-q\right)$ subject to $q \in[0,1]$ if and only if $\frac{\beta Q_{-s}}{q_{s}}-\frac{(1-\beta)\left(Q_{-s}+q_{s}\right)}{1-q_{s}}=0$.

It is now easy to see that $q_{s}=\frac{n-1}{n-\beta} \beta$ does indeed satisfy the preceding equation. Thus $\left(q_{s}\right)$ is an oligopoly equilibrium.

Since $0<\beta<1, \frac{n-1}{n-\beta}$ increases as ' $n$ ' increases and hence $q_{s}=\frac{n-1}{n-\beta} \beta$ increases as $n$ increases. Further, $Q=n \beta \frac{n-1}{n-\beta}$ increases as ' $n$ ' increases. Q.E.D.

Proposition 2: At the unique oligopoly equilibrium the price that each buyer pays for $\mathrm{Y}$ is

$$
\begin{aligned}
& \frac{m(1-\alpha)(n-\beta)}{n(n-1) \beta} \text {. Each buyer consumes } \\
& \left(\alpha, \beta \frac{n}{m} \frac{(n-1)}{n-\beta}\right) \quad \text { and each seller consumes } \\
& \left(\frac{m(1-\alpha)}{n}, \frac{n(1-\beta)}{(n-\beta)}\right) .
\end{aligned}
$$

Proof: Let $\left(q_{s}\right)$ be the unique oligopoly equilibrium. Then we know that the market equilibrium price for $\left(q_{s}\right)$ is $\frac{m(1-\alpha)}{Q}$. From Proposition 1 we get that $Q=n \frac{n-1}{n-\beta} \beta$. Thus the price that each buyer pays for $\mathrm{Y}$ is $\frac{m(1-\alpha)(n-\beta)}{n(n-1) \beta}$.

Since each buyer consumes $\left(\alpha, \frac{Q}{m}\right)$ substituting 
$Q=n \frac{n-1}{n-\beta} \beta$ from Proposition 1, gives the desired consumption bundle of the seller.

Since each seller ' $s$ ' consumes $\left(\frac{m(1-\alpha)}{Q_{-s}+q_{s}} q_{s}, 1-q_{s}\right)$ the desired expression follows from the fact

$q_{s}=\frac{Q}{n}=\frac{n-1}{n-\beta} \beta$ as established in Proposition 1. Q.E.D.

\section{Proposition 3:}

1) Given ' $n$ ' (the number of sellers), the price of $Y$ increases, each buyer is worse off and each seller is better off as ' $m$ ' (the number of buyers) increases.

2) Given ' $m$ ' (the number of buyers), the price of $Y$ decreases, each buyer is better off and each seller is worse off as ' $n$ ' (the number of sellers) increases.

\section{Proof:}

1) Suppose ' $n$ ' remains fixed and ' $m$ ' increases. Then the price of $\mathrm{Y}$ i.e. $\frac{m(1-\alpha)(n-\beta)}{n(n-1) \beta}$ increases. Since the price of $Y$ increases if ' $n$ ' remains fixed and ' $\mathrm{m}$ ' increases whatever is available now was also available earlier to any buyer who was in the market before ' $m$ ' increased. Hence any such buyer is worse off with increase in ' $m$ '. If ' $n$ ' remains fixed and ' $m$ ' increases then there is no change in the consumption of $\mathrm{Y}$ by a seller; however his consumption of $\mathrm{X}$ goes up and so he is better off.

2) Suppose ' $m$ ' remains fixed and ' $n$ ' increases. The price of $Y$ can be written as $\frac{m(1-\alpha)}{n \beta} \frac{n-\beta}{n-1}$.Since $0<\beta<1, \frac{n-\beta}{n-1}$ is greater than 1 and decreases as ' $n$ ' increases. Further $\frac{m(1-\alpha)}{n \beta}$ decreases as ' $n$ ' increases. Thus the price of Y decreases. Since the price of $Y$ decreases whatever was available to a buyer who was in the market before more sellers arrived, continues to be available after the increase in ' $n$ '. Thus any such buyer is better off. If ' $m$ ' remains fixed and ' $n$ ' increases then the quantity of $\mathrm{X}$ consumed by a seller who was in the market before more sellers arrived decreases and since $0<\beta<1, \frac{n-\beta}{n}$ also decreases leading to a decrease in the consumption of Y by any such seller. Hence any such seller is worse off after the arrival of more sellers in the market. Q.E.D.

Suppose the economy is replicated $\mathrm{k}$ times, where $\mathrm{k}$ is some positive integer. Then there are $m k$ buyers each endowed with 1 unit of $\mathrm{X}$ and each having utility function $\mathrm{U}$ and there are $n k$ sellers each endowed with 1 unit of $\mathrm{Y}$ and each having utility function V. Then by replicating the analysis above we can conclude the following.

Proposition 4: Suppose the economy is replicated $k$ times. At the unique oligopoly equilibrium the price that each buyer pays for $\mathrm{Y}$ is $\frac{m(1-\alpha)(k n-\beta)}{n(k n-1) \beta}$. Each buyer consumes $\left(\alpha, \beta \frac{n}{m} \frac{k n-1}{k n-\beta}\right)$ and each seller consumes $\frac{m(1-\alpha)}{n}, \frac{k n(1-\beta)}{k n-\beta}$.

Proposition 4 leads us to the following result.

\section{Proposition 5:}

1) As ' $k$ ' increases the equilibrium price decreases. The sequence of equilibrium prices thus obtained converges to $\frac{m(1-\alpha)}{n \beta}$ which is the price at the competitive equilibrium of the original economy.

2) As ' $k$ ' increases the buyers are better off. The sequence of consumption bundles of the buyers converges to $\left(\alpha, \beta \frac{n}{m}\right)$ which is the consumption bundle of the buyers at the competitive equilibrium of the original economy.

3) As ' $k$ ' increases the sellers are worse off. The sequence of consumption bundles of the sellers converges to $\left(\frac{m(1-\alpha)}{n}, 1-\beta\right)$ which is the consumption bundle of the sellers at the competitive equilibrium of the original economy.

\section{Proof:}

1) The equilibrium price for the $k^{\text {th }}$ replica of the economy is

$$
\begin{aligned}
& \frac{m(1-\alpha)(k n-\beta)}{n(k n-1) \beta}=\left(\frac{m(1-\alpha)}{n \beta}\right)\left(\frac{k n-\beta}{k n-1}\right) \text { Since } 0 \\
& <\beta<1, \frac{k n-\beta}{k n-1}=\frac{n-\frac{\beta}{k}}{n-\frac{1}{k}}>1 \text { and } \frac{k n-\beta}{k n-1} \text { de- }
\end{aligned}
$$

creases as $k$ increases. Hence the price of $\mathrm{Y}$ decreases as $k$ increases. Further the sequence $\left\langle\frac{k n-\beta}{k n-1} / k \in \mathrm{IN}\right\rangle \mid=\left\langle\frac{n-\frac{\beta}{k}}{n-\frac{1}{k}} / k \in \mathrm{IN}\right\rangle \quad$ converges to 1 as $k$ tends to plus infinity. Hence the sequence of prices converges to $\frac{m(1-\alpha)}{n \beta}$.

2) The consumption bundle of a buyer for the $k^{\text {th }}$ rep- 
lica of the economy is $\left(\alpha, \beta \frac{n}{m} \frac{k n-1}{k n-\beta}\right)$. Since $0<$ $\beta<1, \quad 0<\frac{k n-1}{k n-\beta}<1$ and $\frac{k n-1}{k n-\beta}$ increases as $k$ increases. Thus as ' $k$ ' increases the buyers are better off. Further the sequence $\left\langle\frac{k n-1}{k n-\beta} / k \in \mathrm{IN}\right\rangle$ converges to 1 . Thus the sequence of consumption bundle of the buyers converges to $\left(\alpha, \beta \frac{n}{m}\right)$.

3) The consumption bundle of a seller for the $k^{\text {th }}$ replica of the economy is $\left(\frac{m(1-\alpha)}{n}, \frac{k n(1-\beta)}{k n-\beta}\right)$. $\frac{k n(1-\beta)}{k n-\beta}=\frac{1-\beta}{1-\frac{\beta}{k n}}$ decreases as $k$ increases. Thus as ' $k$ ' increases sellers are worse off. Further the sequence

$\left\langle\frac{1-\beta}{1-\frac{\beta}{k n}} / k \in \mathrm{IN}\right\rangle$ converges to $1-\beta$. Thus the sequence of consumption bundles of the buyers converges to $\left(\frac{m(1-\alpha)}{n}, 1-\beta\right)$. Q.E.D.

\section{Conclusions}

While for bilateral oligopoly there are several studies dealing with comparative statics, such is not the case for the kind of (asymmetric) oligopoly that we deal with here. It is true that there is a large literature concerned with oligopoly when the sellers are profit maximizing producers. However when the sellers are traders (as in the case of large fixed cost industries with minimal variable costs, like crude oil) there is very little investigation beyond what has been discussed in [6]. The study of such markets has been the objective of this paper.

The major results obtained in this paper are the following:

1) Given the number of buyers both individual as well as aggregate offers go up as the number of sellers increases. Further, the price of $\mathrm{Y}$ decreases, each buyer is better off and each seller is worse off as the number of sellers increases.

2) Given the number of sellers, the price of $Y$ increases, each buyer is worse off and each seller is better off as the number of buyers increases.

3) As the economy is replicated the equilibrium price decreases. The sequence of equilibrium prices thus obtained converges to the competitive equilibrium price of the original economy.

4) As the economy is replicated the buyers are better off. The sequence of consumption bundles of the buyers converges to the consumption bundle of the buyers at the competitive equilibrium of the original economy.

5) As the economy is replicated the sellers are worse off. The sequence of consumption bundles of the sellers converges to the consumption bundle of the sellers at the competitive equilibrium of the original economy.

The results that we obtain are thus along expected lines in a market economy and economic theory tells us that such phenomena is generally observable under perfect competition. Here we see that these same results are observable under oligopoly as well. This leads us to conclude that simply by observing the behavior of markets and the related entry-exit dynamics we cannot decide that a market is perfectly competitive. In fact the presence of a few sellers and a finite number of buyers points to the greater likelihood of the underlying market structure being oligopolistic.

\section{Acknowledgements}

I would like to thank (without implicating anyone) an anonymous referee of this journal for comments and suggestions which I believe has led to a much better paper.

\section{References}

[1] M. Shubik, "Commodity Money, Oligopoly, Credit and Bankruptcy in a General Equilibrium Model,” Economic Inquiry, Vol. 11, No. 1, March 1973, pp. 24-38.

[2] L. S. Shapley, "Non-Cooperative General Exchange,” In: S. A. Y. Lin, Ed., Theory of Measurement of Economic Externalities, Academic Press, New York, 1976.

[3] L. S. Shapley and M. Shubik: "Trade Using One Commodity as a Means of Payment," Journal of Political Economy, Vol. 85, No. 5, October 1977, pp. 937-968. doi:10.1086/260616

[4] J. J. Gabszewicz and P. Michel, "Oligopoly Equilibrium in Exchange Economies,” In: B. C. Eaton and R. G. Harris, Eds., Trade, Technology and Economics: Essays in Honor of Richard G. Lipsey, Elgar, Cheltenham, 1997.

[5] F. Bloch and H. Ferrer, "Trade Fragmentation and Coordination in Strategic Market Games," Journal of Economic Theory, Vol. 101, No. 1, November 2001, pp. 301316. doi:10.1006/jeth.2000.2730

[6] G. Codognato and J. J. Gabszewicz, "Economie du Cournot-Walras dans une economie d'exchange,” Revue Economique, Vol. 42, No. 6, 1991, pp. 1013-1026. 
[7] R. Amir and F. Bloch: "Comparative Statics in a Simple Class of Strategic Market Games,” Games and Economic Behavior, Vol. 65, No. 1, 2009, pp. 7-24. doi:10.1016/j.geb.2007.09.002

[8] S. Lahiri, "Asymptotic Convergence to Competitive Equilibrium of Oligopoly Equilibria,” 2010. http://papers.ssrn.com/sol3/papers.cfm?abstract_id=1739 486

[9] R. Lahmandi-Ayed, “Oligopoly Equilibria in Exchange Economies: A Limit Theorem,” Economic Theory, Vol. 17, No. 3, 2001, pp. 665-674. doi:10.1007/PL00004122 